\title{
Rate Equation Modelling of Nonlinear Dynamics in Directly Modulated Multiple Quantum Well Laser Diodes
}

\author{
STEPHEN BENNETT $^{\mathrm{a}, *}{ }^{*}$ CHRISTOPHER M. SNOWDEN $^{\mathrm{b}}$ and STAVROS IEZEKIEL $^{\mathrm{b}}$ \\ ${ }^{a}$ Department of Electronic and Electrical Engineering, University College London, London, WCIE 7JE, UK; \\ ${ }^{\mathrm{b}}$ Department of Electronic and Electrical Engineering, University of Leeds, Leeds, LS2 9JT, UK
}

\begin{abstract}
A theoretical (using rate equations) and experimental study of the nonlinear dynamics of a distributed feedback multiple quantum well laser diode is presented. The analysis is performed under direct modulation. Period doubling and period tripling are identified in both the measurements and simulations. Period doubling is found over a wide range of modulation frequencies in the laser. Computational results using rate equations show good agreement with the experimental results.
\end{abstract}

Keywords: MQW laser diodes, rate equations, nonlinear dynamics, period doubling, period tripling

\section{INTRODUCTION}

Multiple Quantum Well (MQW) laser diodes are widely used in many applications because of their superior performance characteristics over bulk laser diodes. These include higher modulation bandwidths and lower threshold currents, both of which are desirable qualities for high-speed optical fibre links. Directly modulated laser diodes are operated under conditions that can lead to a wide variety of nonlinear behaviour, including period doubling [1] and chaos [2]. It is therefore vital to have an accurate model of nonlinear behaviour that is numerically efficient, and for the most part rate equations are used by most workers. However, in contrast to a large volume of work using rate equations for nonlinear modelling of bulk structures, little such work exists for MQW lasers. This paper presents a detailed theoretical and experimental study of the nonlinear dynamics of an InGaAsP/InGaAs MQW $\lambda / 4$ shifted Distributed Feedback (DFB) laser diode with 16 quantum wells [3] under direct modulation.

\footnotetext{
* Corresponding author.
} 


\section{MQW LASER DIODE MODEL}

To account for the carrier dynamics in quantum well lasers, a rate equation model based on that proposed by Nagarajan et al. [4] is used in our numerical simulations. The rate equations for the carrier density in the quantum wells $(N)$ and the barriers $\left(N_{B}\right)$ and the photon density in the optical cavity $(S)$ can be written as,

$$
\begin{gathered}
\frac{d N_{B}}{d t}=\frac{\Gamma_{\mathrm{q}} I}{e V}-\frac{N_{\mathrm{B}}}{\tau_{\mathrm{c}}}+\frac{\Gamma_{\mathrm{q}} N}{\tau_{\mathrm{e}}} \\
\frac{d N}{d t}=\frac{N_{B}}{\Gamma_{\mathrm{q}} \tau_{\mathrm{c}}}-N\left[\frac{1}{\tau_{\mathrm{n}}}+\frac{1}{\tau_{\mathrm{e}}}\right]-v_{\mathrm{g}} G(N, S) S \\
\frac{d S}{d t}=\left[\Gamma v_{\mathrm{g}} G(N, S)-\frac{1}{\tau_{\mathrm{p}}}\right] S+\Gamma \beta B N^{2}
\end{gathered}
$$

where $\Gamma_{\mathrm{q}}$ is the fraction of the MQW region filled by the quantum wells, $N_{B} / \tau_{\mathrm{c}}$ is the loss rate of carriers from the barriers to the quantum wells and $N / \tau_{\mathrm{e}}$ is the loss rate of carriers from the quantum wells to the barriers. $\tau_{\mathrm{p}}$ is the photon lifetime, $V$ is the active region volume, $\Gamma$ is the mode confinement factor, and $v_{\mathrm{g}}$ is the group velocity. The dependence of the carrier lifetime $\left(\tau_{\mathbf{n}}\right)$ on $N$ is modelled as,

$$
\frac{1}{\tau_{\mathrm{n}}}=A+B N+C N^{2}
$$

where the terms $B N$ and $C N^{2}$ model bimolecular and Auger recombination respectively. The output power per facet $P$ is related to $S$ via,

$$
P=\frac{S V h v v_{\mathrm{g}}}{2 \Gamma L} \ln \left(\frac{1}{R}\right)
$$

where $R$ is the facet reflectivity $(\approx 0.32)$ and $L$ is the cavity length. The injection current $(I)$ can be expressed $I=I_{\mathrm{DC}}+I_{\mathrm{RF}} \sin (2 \pi f t)$, where $I_{\mathrm{DC}}$ is the bias current, $I_{\mathrm{RF}}$ is modulation current amplitude and $f$ is the modulation frequency. Using an isolator between the microwave source and the laser to minimise impedance mismatch problems enables $I_{\mathrm{RF}}$ to be approximated, $I_{\mathrm{RF}} \approx \sqrt{2 P_{\mathrm{RF}} / Z_{0}}$
$\Gamma_{\mathrm{L}}-1 \mid$, where $P_{\mathrm{RF}}$ is the incident RF input power, $\Gamma_{\mathrm{L}}$ is the reflection coefficient of the laser and $Z_{0}$ is the characteristic impedance. The quantum wells have one conduction subband so that the relationship between the carrier density and the junction voltage $V_{\mathrm{j}}$ can be approximated [5],

$$
N \approx k T \frac{m_{\mathrm{c}}}{\pi \hbar^{2} L_{\mathrm{z}}} \ln \left[1+\exp \left(\frac{e V_{\mathrm{j}}-E_{\mathrm{ph}}}{k T}\right)\right]
$$

where $m_{\mathrm{c}}$ is the electron effective mass, $L_{\mathrm{z}}$ is the quantum well width and $E_{\mathrm{ph}}$ is the photon energy. The MQW material gain $G(N, S)$ is modelled by [6]

$$
G(N, S)=\frac{G_{0}}{(1+\varepsilon S)} \ln \frac{N}{N_{0}}
$$

where $\varepsilon$ is the nonlinear gain coefficient, $N_{0}$ is the transparent carrier density and $G_{0}$ is a constant dependant on well structure.

It is known that the fraction of the spontaneous emission coupled into the lasing mode ( $\beta$ factor) plays a large part in determining the nonlinear dynamics of laser diodes [2,7-9] and it has recently been found that bulk DFB laser diodes can exhibit a reduced $\beta$ factor [2] compared to their FP counterparts $\left(8 \times 10^{-7}\right.$ compared to $10^{-5}$ in typical FP lasers). The value of $\beta$ used in our simulations was therefore measured using the same technique described in [2]. Other parameters which were not material or dimensional in nature were

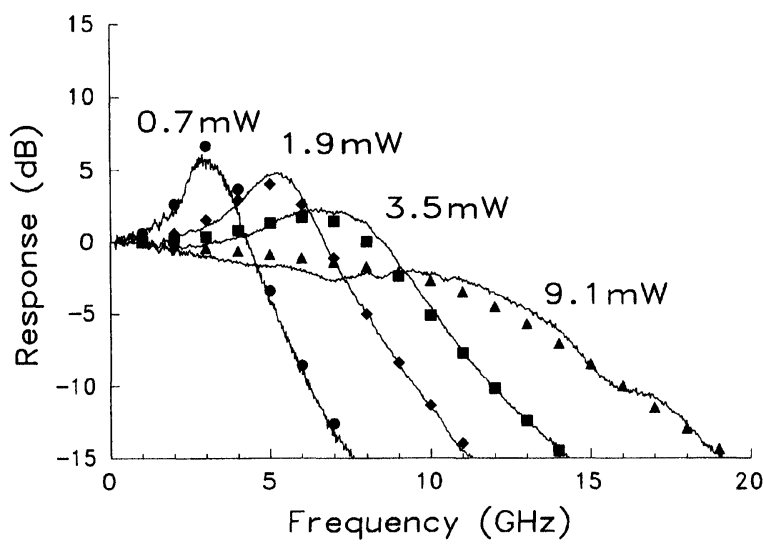

FIGURE 1 Measured (solid lines) and simulated (points) small-signal modulation frequency response of the laser with increasing output power. 
extracted from the small-signal measurements shown in Figure 1. The parameter values used in the simulations were: $V=5.1 \times 10^{-17} \mathrm{~m}^{3}, \Gamma=0.22$, $\Gamma_{q}=0.66, \tau_{\mathrm{p}}=1.3 \mathrm{ps}, \beta=10^{-6}, G_{0}=141107 \mathrm{~m}^{-1}$, $N_{0}=2.41 \times 10^{24} \mathrm{~m}^{-3}, \varepsilon=3.24 \times 10^{-23} \mathrm{~m}^{3}, A=10^{8}$ $\mathrm{s}^{-1}, \quad B=10^{-16} \mathrm{~m}^{3} \mathrm{~s}^{-1}, C=3 \times 10^{-41} \mathrm{~m}^{6} \mathrm{~s}^{-1}$, $\tau_{\mathrm{c}}=20 \mathrm{ps}$, and $\tau_{\mathrm{e}}=191 \mathrm{ps}$. Simulations were performed by solving (1)-(3) using a fourth order Runge-Kutta scheme.

\section{NONLINEAR DYNAMIC ANALYSIS}

\subsection{Results}

So as to study the nonlinear dynamics resulting from direct modulation of the laser alone, optical feedback into the laser was minimised by the use of an optical isolator. The experimental setup used to analyse the nonlinear dynamics of the lasers is shown in Figure 2. The incident RF input power to the laser $\left(P_{\mathrm{RF}}\right)$ was monitored through a directional coupler and the problem of laser mismatch to $50 \Omega$ was minimised through the use of a microwave isolator.

For a range of output power levels* $\left(P_{\text {out }}\right)$ and modulation frequencies $(f), P_{\mathrm{RF}}$ was swept between -5 and $22 \mathrm{dBm}$ and the regions where nonlinear behaviour occurred recorded. These regions are shown in Figure 3 along with the simulated results. There were both regions of period doubling and period tripling. Period tripling occurred when the laser was biased with

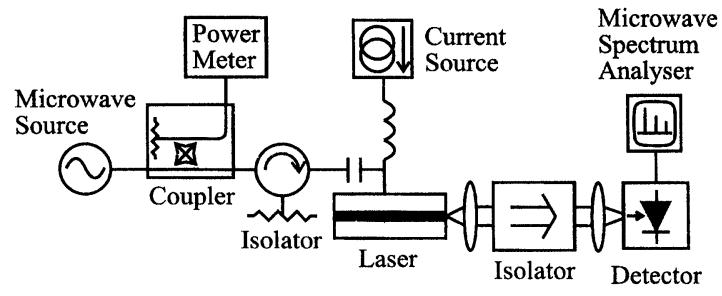

FIGURE 2 Experimental setup used to investigate the nonlinear dynamics of the laser.
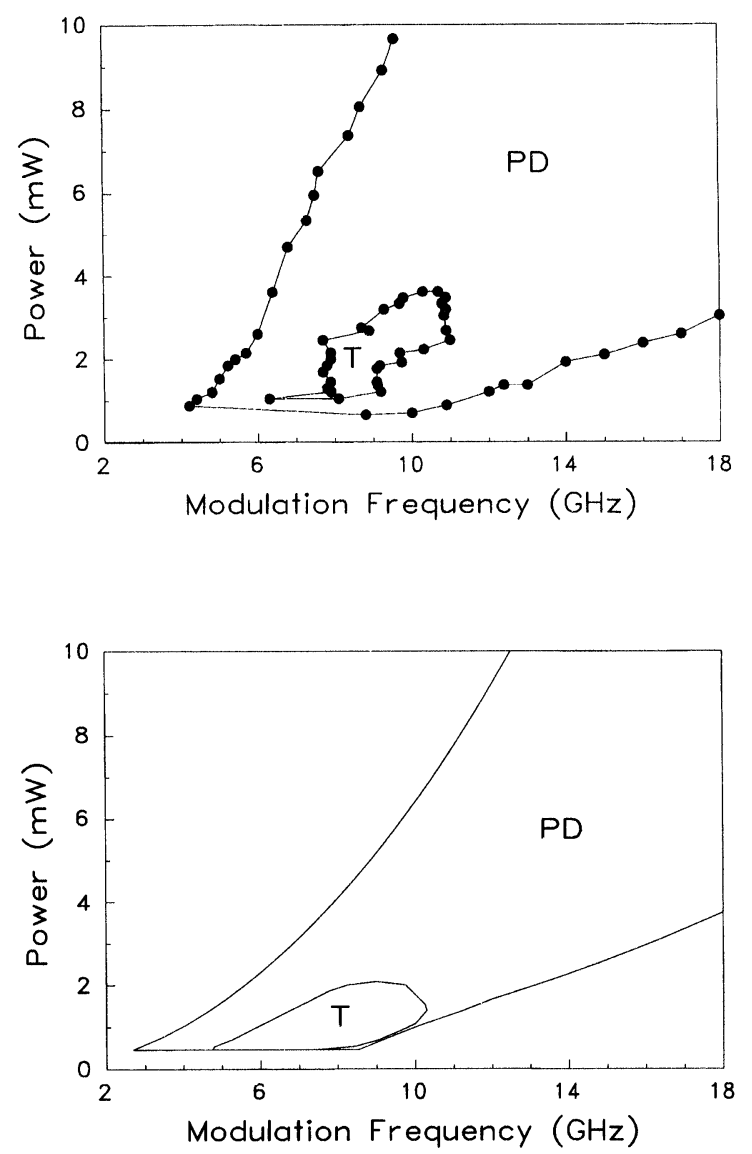

FIGURE 3 Regions of where nonlinear behaviour were recorded for (a) the measured laser, (b) the simulated laser. PD denotes the regions where period doubling were recorded and $\mathrm{T}$ the region where period tripling was observed.

$P_{\text {out }}$ levels of between $0.7 \mathrm{~mW}$ and $3.6 \mathrm{~mW}$. In the region where period tripling occurred, as $P_{\mathrm{RF}}$ was increased, the route to period tripling was always via period doubling. Figure 4 shows the measured and simulated output frequency spectrum of the laser under conditions of single period, period doubling and period tripling behaviour.

There were features of the period doubling which were common to both simulated and measured lasers. Namely the upper and lower modulation frequency limits of the period doubling regions seemed to follow one and two times

* Corresponding to a range of bias currents. 
the relaxation frequency of the laser $\left(f_{\mathrm{R}}\right)$ respectively. For a fixed ratio of modulation frequency to relaxation frequency the value of $P_{\mathrm{RF}}$ required to produce period doubling increased almost linearly with $P_{\text {out }}$. These features are illustrated in Figure 5 where the calculated values of $P_{\mathrm{RF}}$ required for period doubling to occur in the laser are displayed versus $P_{\text {out }}$ and the modulation frequency normalised to $f_{\mathrm{R}}$. The measured and simulated values of $P_{\mathrm{RF}}$ required for period doubling to occur in the laser versus $P_{\text {out }}$ are displayed in Figure 6. When obtaining the data displayed in Figure 6 the modulation frequency was adjusted at each $P_{\text {out }}$ to keep the ratio of modulation frequency to relaxation frequency constant, which for Figure 6 was 1.5. It is expected that this relationship is due to the corresponding increase in relaxation damping

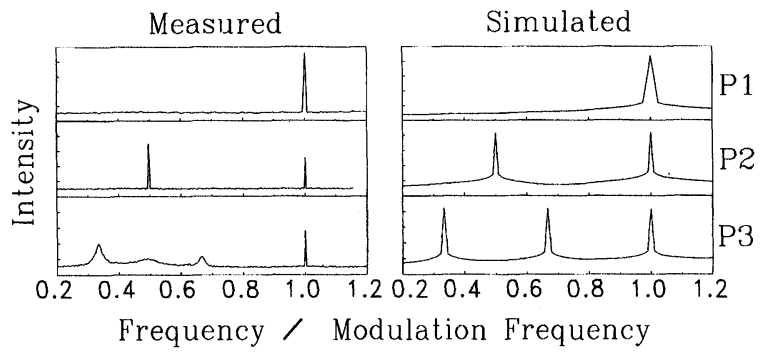

FIGURE 4 Measured and simulated frequency spectrum of the laser under conditions of single period (P1), period doubling (P2) and period tripling (P3) behaviour.

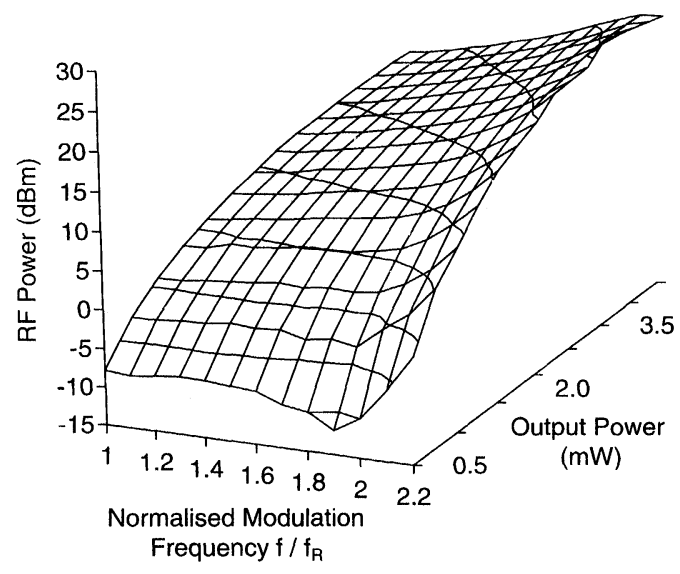

FIGURE 5 Three dimensional plot displaying the calculated values of $P_{\mathrm{RF}}$ required for period doubling to occur in the laser versus $P_{\text {out }}$ and the modulation frequency normalised to $f_{\mathrm{R}}$.

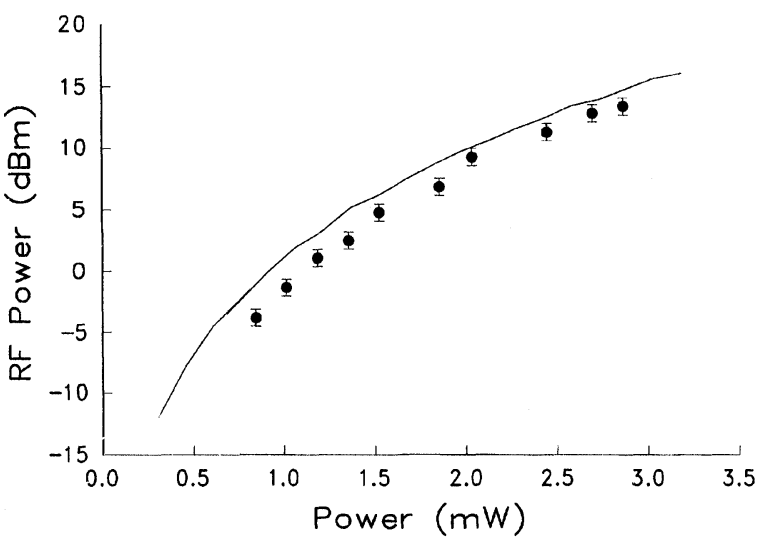

FIGURE 6 Measured (points) and simulated (solid line) RF input power required to produce period doubling versus output power per facet. The modulation frequency to relaxation frequency ratio is kept constant at 1.5.

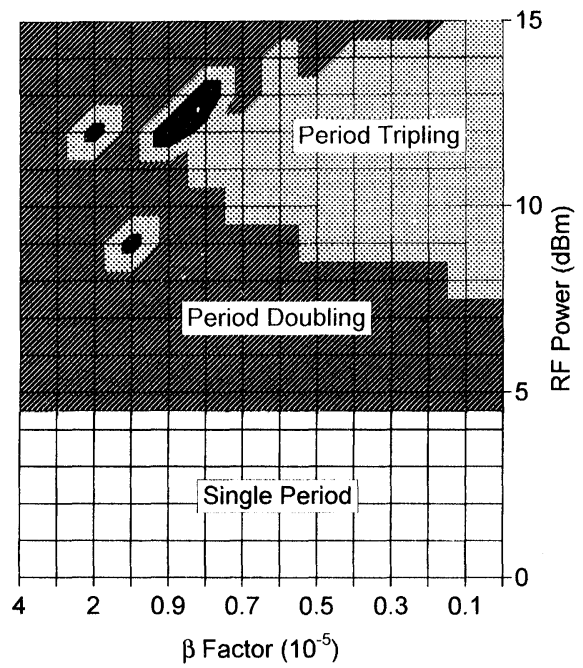

FIGURE 7 Simulated dependence of nonlinear behaviour on $P_{\mathrm{RF}}$ and $\beta$ in the laser. Simulations were performed with a modulation frequency of $6 \mathrm{GHz}$ and $P_{\text {out }}$ set at $0.6 \mathrm{~mW}$. The black areas in the diagram indicate regions of higher order bifurcations.

with power level which is known to be an important factor in characterising the nonlinear dynamics of laser diodes [9].

Liu et al. [2] suggest that the promotion of period tripling in their bulk DFB laser was due to the unusually low value of $\beta$ measured in their laser. To investigate whether this was the case in our MQW DFB laser we analysed the effect of variations in $\beta$ 
on our simulation results. Figure 7 shows the regions of nonlinear behaviour as $\beta$ and $P_{\mathrm{RF}}$ are varied. It is clear that as $\beta$ is reduced the period tripling behaviour becomes more prominent. It would therefore seem likely that the observed period tripling in our DFB laser can be attributed to the low value of $\beta$ measured in this laser compared to that measured in typical Fabry Perot lasers.

\section{CONCLUSION}

In summary, we have performed a detailed theoretical (using rate equations) and experimental analysis of the nonlinear dynamics of a MQW DFB laser diode under direct modulation. We observed period doubling and period tripling in both measurements and simulations. Period doubling was evident over a wide range of modulation frequencies. Computational results using rate equations show good agreement with the experimental results.

\section{Acknowledgements}

The authors would like to acknowledge Dr. L. D. Westbrook and Dr. I. F. Lealman of British Telecom Research Laboratories for informative discussions and the supply of sample lasers.

\section{References}

[1] Hemery, E., Chusseau, L. and Lourtioz, J. M. "Dynamic behaviors of semiconductor lasers under strong sinusoidal current modulation: Modeling and experiments at 1.3 нm”, IEEE J. Quantum Electron., 26, 633-641, April 1990.

[2] Liu, H. F. and Ngai, W. F. "Nonlinear dynamics of a directly modulated $1.55 \mu \mathrm{m}$ InGaAsP distributed feedback semiconductor laser", IEEE J. Quantum Electron., 29, 1668 - 1675, June 1993

[3] Lealman, I. F., Harlow, W. F. and Perrin, S. D. "Effects of $\mathrm{Zn}$ doping on modulation bandwidth of $1.55 \mu \mathrm{m}$ InGaAs/InGaAsP multiquantum well DFB lasers", Electron. Lett., 29, 1197-1198, June 1993.

[4] Nagarajan, R., Ishikawa, M., Fukushima, T., Geels, R. S. and Bowers, J. E. "High speed quantum-well lasers and carrier transport effects", IEEE J. Quantum Electron., 28, 1990 - 2007, Oct. 1992
[5] Seltzer, C. P., Westbrook, L. D. and Wickes, H. J. (1995). "The 'Gain-Lever' effect in InGaAsP/InP multiple quantum well lasers", IEEE J. Lightwave Technol., 13(2), 283-289.

[6] McIlroy, P. W. A., Kurobe, A. and Uematsu. "Analysis and application of theoretical gain curves to the design of multi-quantum-well lasers", IEEE J. Quantum Electron., 21, 1958-1963, Dec. 1985.

[7] Lee, C. H., Yoon, T. H. and Shin, S. Y. "Period doubling and chaos in a directly modulated laser diode", Appl. Phys. Lett., 46, 95-97, Jan. 1985.

[8] Hori, Y., Serizawa, H. and Sato, H. "Chaos in a directly modulated semiconductor laser", J. Opt. Soc. Amer. B., 5, 1128-1133, May 1988.

[9] Yoon, T. H., Lèe, C. H. and Shin, S. Y. "Perturbation analysis of bistability and period doubling bifurcations in directly-modulated laser diodes", IEEE J. Quantum Electron., 25, 1993-2000, Sept. 1989.

\section{Authors' Biographies}

Stephen Bennett was born in Blackpool, England, in 1971. He received the B.Eng. (Honours) degree in electronic and electrical engineering from the University of Leeds in 1993. After graduating he joined the Microwave and Terahertz Technology Group at the University of Leeds where he has been working towards the Ph.D. degree on the high frequency nonlinear dynamics of semiconductor lasers. Since 1996 he has worked as a Research Fellow within the Microwave Optoelectronics group at University College London. His research interests include semiconductor laser dynamics, soliton control, optical comb generation and MQW saturable absorption.

Christopher M. Snowden received the B.Sc., M.Sc. and Ph.D. degrees from the University of Leeds. After graduating in 1977 he worked as an Applications Engineer for Mullard, Mitcham. His Ph.D. studies were conducted in association with Racal-MESL and were concerned with the largesignal characterisation of MESFET microwave oscillators. In 1982 he was appointed Lecturer in the Department of Electronics at the University of York. He joined the Microwave Solid State Group in the Department of Electrical and Electronic Engineering at the University of Leeds in 1983 . He now holds the Chair of Microwave Engineering in the Microwave and Terahertz Technology Research Group and is also currently Head of the 
Department of Electronic and Electrical Engineering. During 1987 he was a Visiting Research Associate at the California Institute of Technology. He has been a Consultant to M/A-COM Inc., Corporate Research and Development since 1989, where he was on sabbatical leave during the period 1990-91. During this year he represented M/ACOM as Senior Staff Scientist. He was Chairman of the 1995 international Microwaves and RF Conference. He is a Member of the MIT Electromagnetics Academy. He is also a Top Scientist at the International Research Centre for Telecommunications-Transmission and Radar, Delft University of Technology, Netherlands.

Professor Snowden is a Fellow of the IEEE and a Fellow of the IEE. He is a Distinguished Lecturer (1996/7) for the IEEE (Electron Devices Society). He is co-Chairman of the MTT-1 Committee and a Member of the 1997 IEEE MTT-S Technical
Program Committee. His main research interests include compound semiconductor device modelling, microwave, terahertz and optical nonlinear subsystem design and advanced semiconductor devices. He has written 7 books and over 190 papers.

Stavros Iezekiel was born in Coventry, England, in 1966. He received the B.Eng. and Ph.D. degrees in electronic and electrical engineering from the University of Leeds in 1987 and 1991, respectively. From 1991 to 1993, he worked as a Research Fellow developing hybrid optoelectronic packaging systems for M/A-Com Corporate Research and Development Centre. In 1993, he was appointed as a Lecturer in high-frequency analogue electronics at the University of Leeds. His research interests include high-speed semiconductor laser diode modelling, optoelectronic packaging, nonlinear systems, and microwave-optoelectronic subsystem design. 

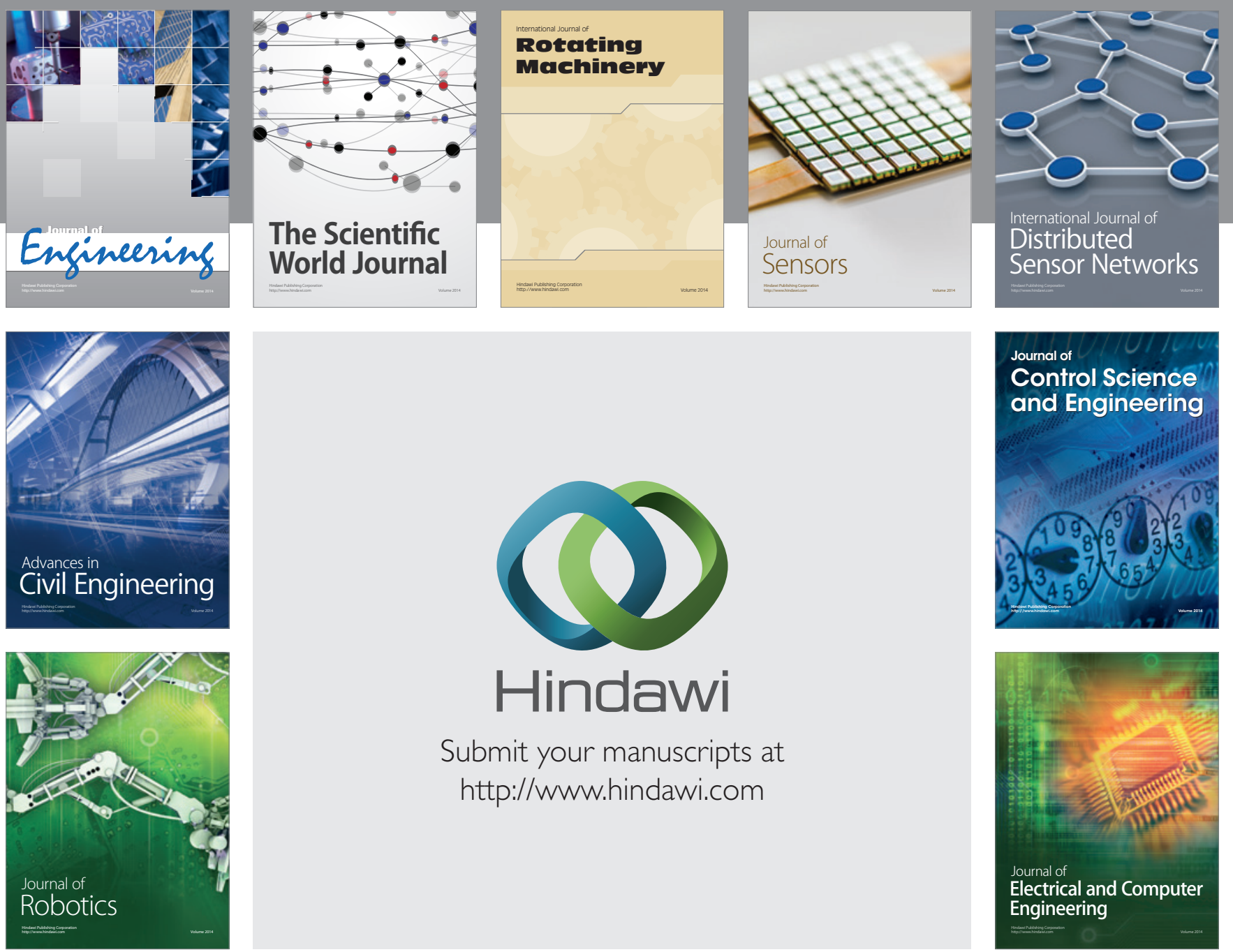

Submit your manuscripts at

http://www.hindawi.com
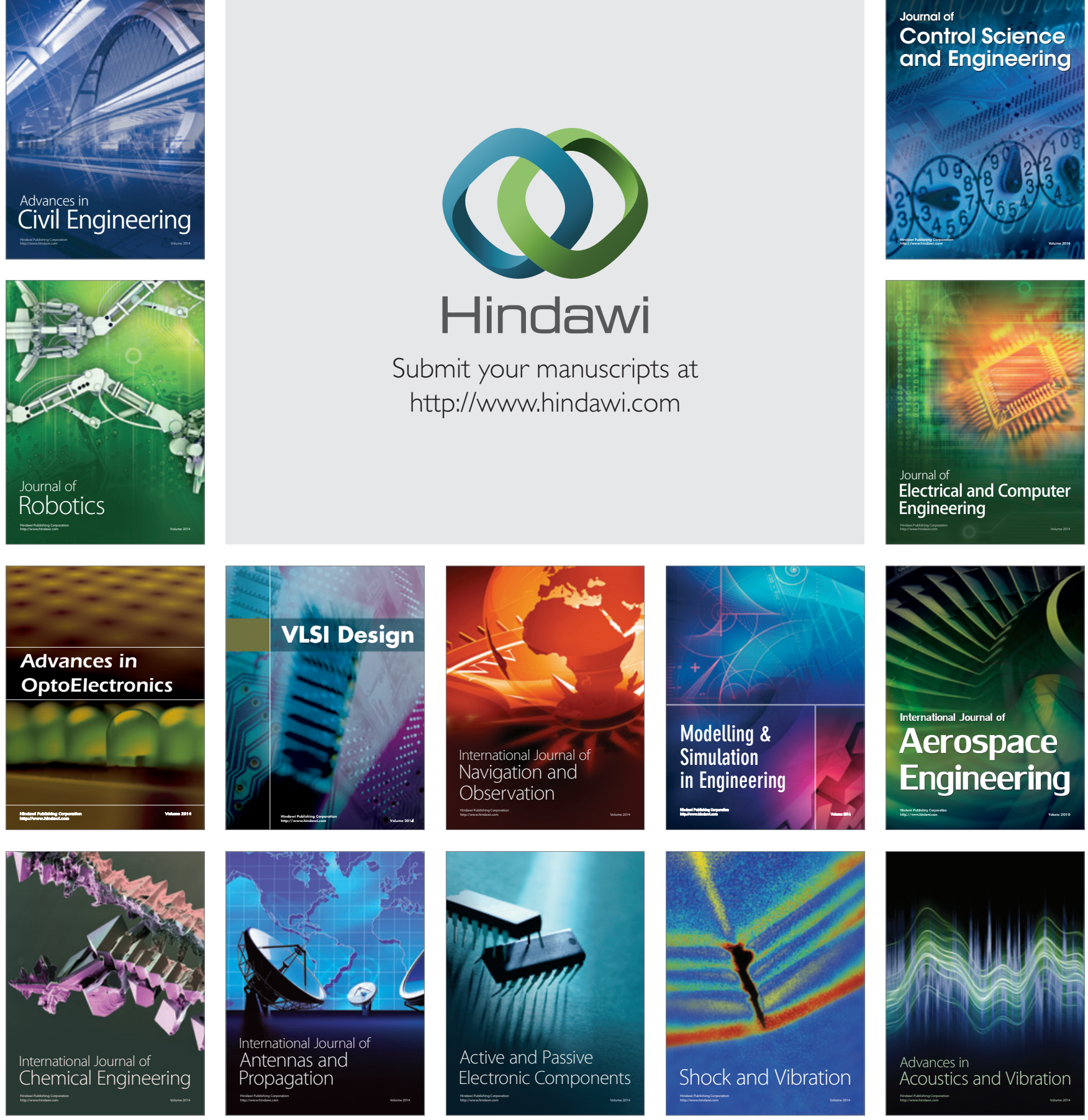